\title{
Validación en México del inventario de ajuste de Weinberger (WAI)
}

\author{
Tania Romo González, ${ }^{1}$ Claudia Beatriz Enríquez-Hernández, ${ }^{2}$ María del Rocío Hernández Pozo, ${ }^{3}$ \\ María Elena Ruiz Montalvo, ${ }^{2}$ Rosa Lilia Castillo, ${ }^{4}$ Yamilet Ehrenzweig Sánchez, ${ }^{4}$ \\ María Luisa Marván, ${ }^{4}$ Carlos Larralde ${ }^{5}$
}

Artículo original

\section{SUMMARY}

\section{Introduction}

The inability to control emotional outbursts and the excessive inhibition of emotions are associated with a variety of personal and interpersonal problems, psychological disorders and health status. Weinberger developed an inventory to assesses two central aspects of socio-emotional adjustment: the subjective experience of distress and restraint. As the inventory was in English, we decided to translate it into Spanish and validated it in a population of Spanish-speaking Mexicans.

\section{Method}

The revised Spanish WAI was applied to 452 participants using the same Likert scale used in the English WAl, in which each answer was given a score of five points. The participants were adults (over 18 years) of either sex with a minimum educational level of high school.

\section{Results}

After analyzing the discriminatory power of the questions by the method of extreme groups and factor analysis by principal components analysis, the Spanish WAI was composed of 44 items, which were divided into four factors: 1 . Restraint, which grouped together a total of 19 questions, which explained $18.68 \%$ of the variance, 2 . Subjective experience of distress, consisting of 12 questions, which accounted for $10.84 \%$ of the variance, 3 . Defensiveness, consisting of seven questions that explained $7.10 \%$ of the variance, and 4 . Consideration for Others, which grouped six questions that explained $6.54 \%$ of the variance. Finally, we assessed the reliability of the questionnaire using Cronbach's alpha as follows: Restraint 0.89, Subjective experience of distress 0.84 , Defensiveness 0.69 , and Consideration for others 0.74 .

\section{Conclusions}

Weinberger's Inventory is a useful instrument to assess restraint as an important aspect of emotional distress of individuals, and to measure the magnitude of restraint in a population, and thus allowing preventive and therapeutic actions in cases that deserve it.

Key words: Spanish validation, Weinberger inventory, distress, emotional restraint, socio-emotional adjustment, Spanish-speaking.

\section{RESUMEN}

\section{Introducción}

La incapacidad para aprender a regular los arranques emocionales y la inhibición excesiva de emociones se asocian con una gran variedad de problemáticas personales e interpersonales, así como con alteraciones psicológicas y de salud. Weinberger desarrolló un inventario que evalúa dos aspectos centrales del ajuste socioemocional: la experiencia subjetiva de distrés y la contención emocional. El inventario sólo existía en inglés, por lo que resolvimos traducirlo al español y validarlo en una población de hispanoparlantes mexicanos.

\section{Método}

El cuestionario traducido y revisado se aplicó a 452 participantes, respetando la escala Likert del cuestionario original de cinco puntos. Los participantes fueron personas adultas (mayores de 18 años) sin distinción de sexo que tenían un nivel de escolaridad mínimo de secundaria.

\section{Resultados}

Tras el análisis de la capacidad discriminatoria de los reactivos por el método de grupos extremos y el análisis factorial por el método de componentes principales, el cuestionario quedó conformado por 44 reactivos, agrupados en cuatro factores: 1 . Dominio de sí mismo con un total de 19 preguntas que explicaron $18.68 \%$ de la varianza, 2 . Experiencia subjetiva de distrés, compuesto por 12 preguntas que explicaron $10.84 \%$ de la varianza, 3. Defensividad, el cual constó de siete preguntas que explicaron $7.10 \%$ de la varianza y 4 . Consideración hacia los demás, que agrupó seis preguntas que explicaron $6.54 \%$ de la varianza. Finalmente, se evaluó la confiabilidad del cuestionario utilizando el alpha de Cronbach, y quedó como sigue: Dominio de sí mismo 0.89, Experiencia subjetiva de distrés 0.84 , Defensividad 0.69 y Consideración hacia los demás 0.74 .

\section{Conclusiones}

El Inventario de Weinberger es una herramienta útil para evaluar el ajuste socioemocional, así como para determinar la magnitud de la inhibición emocional en una población, abordar su prevención e instrumentar acciones para la atención de los casos que lo ameriten.

Palabras clave: Validación, inventario de Weinberger, distrés, contención emocional, ajuste socioemocional, propiedades psicométricas, hispanoparlantes.

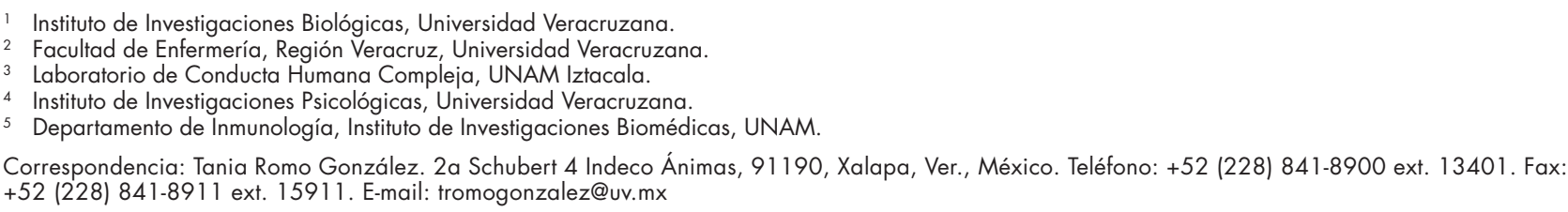




\section{INTRODUCCIÓN}

Desde los primeros meses de vida, los bebés expresan sus reacciones emocionales a quienes les rodean; posteriormente aprenden que tales exhibiciones tienen tanto costos interpersonales como ventajas. Poco a poco empiezan a ocultar en diversos grados sus emociones a los demás, y comienzan a utilizar estrategias cognitivas que alteran su comportamiento. ${ }^{1-6}$ Por lo tanto, en la edad adulta existe poca correlación entre la experimentación de una emoción y su expresión. ${ }^{7}$ De hecho, como los individuos tienen al crecer cada vez más interacciones sociales fuera de la familia, la necesidad de modular sus impulsos emocionales y su comportamiento se convierte en un requisito fundamental de la madurez y el funcionamiento humano efectivo. ${ }^{8}$

No es sorprendente que la incapacidad para aprender a regular los arranques emocionales y la inhibición excesiva de emociones predigan una gran variedad de problemáticas a lo largo de la vida, como dificultades interpersonales, alteraciones psicológicas y de salud, ${ }^{9-12}$ mientras que las personas bien adaptadas o con parámetros de contención emocional saludables presentan poco distrés subjetivo y altos niveles de espontaneidad, asertividad y libre expresión. ${ }^{13,14}$ Por ejemplo, los niveles anormales de distrés y contención son relativos a varios problemas, incluyendo los comportamientos delincuenciales, los trastornos de estrés postraumático entre delincuentes y el abuso de alcohol entre estudiantes universitarios. ${ }^{15}$

Los instrumentos que utilizan medidas estándar de ansiedad, depresión o ira suponen que un informe de niveles bajos sería óptimo. Sin embargo, a menudo no es posible detectar la contención emocional con medidas indirectas. ${ }^{16}$ En estudios de laboratorio se ha visto que las personas que se contienen, aunque reportan niveles muy bajos de distrés subjetivo, exhiben niveles de reactividad al estrés, iguales o mayores que las personas que admiten experimentar un distrés considerable. ${ }^{13}$ Incluso, aunque las personas que se contienen informan no experimentar emociones, los cambios en su ritmo cardiaco, presión arterial, tensión muscular, actividad de las glándulas sudoríparas, expresiones faciales, patrones paralingüísticos y tiempos de reacción sugieren que exhiben una reactividad más elevada que los individuos que no se contienen. ${ }^{17-19}$ Además, la inhibición emocional también está implicada en la exacerbación de una variedad de problemas de salud, incluyendo el asma, el cáncer, la hipertensión y el mal funcionamiento del sistema inmune. ${ }^{13,20}$ Por lo tanto, se ha concluido que una persona que inhibe sus emociones no necesariamente presenta una situación deseable, desde el punto de vista del comportamiento saludable. Sin embargo, la naturaleza de la estructura y organización de una persona que inhibe sus emociones es limitada. ${ }^{21,22}$ Esto se debe a que se han acumulado resultados de las dimensiones que integran la inhibición emocional de forma aislada (i.e., depresión, impulsividad, asertividad), sin tomar en cuenta cómo aumentan estos atributos la probabilidad de comportamientos patogénicos o inmunogénicos. ${ }^{23-27}$

Weinberger $^{27}$ ha empleado una perspectiva integral para desarrollar un inventario que evalúa el ajuste socioemocional, el cual tiene dos dimensiones: a) La dimensión afectiva o experiencia subjetiva del distrés, que proporciona una medida general de las tendencias de los individuos a sentirse insatisfechos con ellos mismos. De esta manera, la predisposición a la ansiedad o a la depresión, la baja autoestima y el bajo bienestar se definen operacionalmente como subtipos de distrés. b) La segunda dimensión mide la contención (es decir, el dominio de sí mismo) y abarca variables socioemocionales relacionadas con la socialización y el autocontrol. Se refiere a la supresión de los deseos egoístas a corto plazo por el interés hacia los demás a largo plazo. Así, la contención es superpuesta ${ }^{25}$ a las tendencias para inhibir la conducta agresiva para ejercer el control de los impulsos, para actuar de forma responsable y para ser considerado con los demás.

El distrés y la contención pueden ubicarse también en el contexto de los "Cinco Grandes" factores de la personalidad $^{26,27}$ y de los tres factores modelo de Eysenck y Tellegen. ${ }^{24,28}$ Además, aunque en los detalles difieren considerablemente, la conceptualización de distrés y contención como dos dimensiones amplias y relativamente estables de ajuste son paralelas a la construcción de la ego-resiliencia y del ego-control. ${ }^{29}$

En este sentido, el Inventario de Ajuste de Weinberger (WAI) ${ }^{13,28,30,31}$ es un buen instrumento para medir el ajuste socioemocional, así como las dimensiones y subdimensiones de la personalidad de manera eficiente y confiable. Consta de 84 reactivos y se compone de tres factores: Distrés (ansiedad, depresión, baja autoestima y bajo bienestar), Contención (represión de la agresión, control de impulsos, consideración hacia los otros y responsabilidad) y Defensividad (actitud defensiva, negación del distrés). La estructura factorial de las escalas de distrés y contención se conservan desde la adolescencia temprana hasta la edad avanzada, tanto en casos clínicos como en personas normales. ${ }^{14,32-35}$ Las tres escalas han demostrado tener consistencia interna superior a 0.85 en diversas muestras y una confiabilidad por encima de 0.7 mediante la prueba test-retest en una muestra de adolescentes tempranos y siete meses después. Además, al compararlo con los otros seis instrumentos existentes de represión, ha sido el cuestionario con mejores propiedades psicométricas dentro de los seis instrumentos existentes de ajuste socioemocional (la Escala de Represión y Sensibilización de Byre, la Escala de Afrontamiento Represivo de Weinberger, la Escala de Monitoreo y Embotamiento de Miller, el Cuestionario de Autoengaño de Sackeim y Gur y el Cuestionario de Autoengaño de Paulus). ${ }^{36}$

Con el uso de este instrumento se ha demostrado la relación entre la expresividad emocional disminuida y algunos padecimientos como el cáncer. Por ejemplo, la represión 
(baja ansiedad y alta defensividad) y la supresión (retención deliberada de la expresión de emociones negativas) se asocian con una progresión más rápida del cáncer de mama y con una expectativa de sobrevida más corta. ${ }^{37}$ De hecho, la supresión y la represión se han considerado como variables psicológicas que afectan notoriamente el ajuste psicosocial en personas con cáncer, pudiendo ser predictores en la incidencia de cáncer de mama ${ }^{38}$ y en el pronóstico de una progresión más rápida del mismo. ${ }^{39}$

Por todas estas características y virtudes del WAI es que el objetivo del presente estudio fue traducirlo al español y validarlo en una muestra de adultos mexicanos.

\section{MATERIAL Y MÉTODO \\ Participantes}

Se estudió una muestra de 452 sujetos, de los cuales 182 fueron hombres, 265 mujeres y cinco no especificaron el sexo. Sus edades oscilaron entre 18 y 70 años de edad (media 27.93, SD 11.03). Todos los participantes vivían en las ciudades de Xalapa o Veracruz y tenían un nivel socioeconómico medio o medio bajo. En cuanto a la escolaridad, el 70\% tenía secundaria o preparatoria y el 30\% educación superior. El número de participantes se eligió para cumplir con el criterio de por lo menos cinco sujetos por cada reactivo del cuestionario original. ${ }^{40,41}$

Los criterios de inclusión fueron: 1. personas adultas (mayores de 18 años), 2. con un nivel de escolaridad mínimo de secundaria, lo que garantiza una buena comprensión del cuestionario y 3. participación voluntaria e informada.

Los participantes fueron contactados ya sea en sus lugares de trabajo o en sus casas por medio de redes personales. Se les solicitó su participación aclarándoseles que el cuestionario era anónimo y que los resultados únicamente se usarían para esta investigación. Cuando una persona aceptaba participar, se acordaba un lugar y una fecha para que contestara el cuestionario. El 90\% de las personas a las que se les solicitó participar aceptaron contestarlo.

\section{Instrumento}

El Inventario de Ajuste de Weinberger evalúa la capacidad general de ajuste socioemocional. Consta de 84 reactivos evaluados en una escala Likert de cinco puntos. En la primera parte del cuestionario (del reactivo 1 al 45) las opciones de la escala son: 1 . falso, 2 . más o menos falso, 3 . no estoy seguro, 4 . más o menos verdadero y 5 . verdadero. En la segunda parte (del reactivo 46 al 84), las opciones son: 1 . casi nunca o nunca, 2. poco frecuente, 3 . algunas veces, 4 . frecuentemente y 5. siempre o casi siempre.

Se compone de tres factores: 1. Distrés (dividido en subescalas de Ansiedad, Depresión, Baja autoestima y Bajo bienestar), 2. Contención o Dominio de sí mismo (dividido en las subescalas de Supresión de la agresión, Control de los impulsos, Consideración hacia los demás y Responsabilidad) y 3. Defensividad (dividido en las subescalas de Represión-defensividad y Negación del distrés).

\section{Procedimiento}

El cuestionario original se tradujo al español y luego de nueva cuenta al inglés para comparar su correspondencia con el original. A continuación se solicitó a cinco expertos en el tema evaluar la redacción del instrumento para que todos los reactivos fueran comprendidos. Posteriormente se realizó un estudio piloto con 10 personas, a quienes se les pidió que respondieran todas las preguntas y al finalizar comentaran si alguna de ellas les pareció confusa o difícil de comprender. Consecuentemente se hicieron algunas modificaciones y se volvió a hacer otro estudio piloto en el que los 15 sujetos participantes dijeron haber entendido bien todos los reactivos.

El cuestionario revisado se aplicó en los meses de enero y febrero del 2012 a los 452 participantes, respetando la escala Likert del cuestionario original, de tal manera que a cada reactivo se le otorgó una calificación del 1 al 5. El encuestador aclaró que no existían respuestas correctas ni incorrectas.

\section{RESULTADOS}

Se realizó un análisis de la capacidad discriminatoria de los reactivos por medio del método de grupos extremos, comparando los puntajes totales del $27 \%$ de los sujetos con los puntajes más elevados y el $27 \%$ de los sujetos con los puntajes más bajos. ${ }^{40}$ Para esta comparación se utilizó la prueba $t$ de Student para muestras independientes y se eliminaron 22 reactivos por no alcanzar el nivel de significancia estadística $(\mathrm{p}<0.05)$.

Posteriormente se realizó un análisis factorial por medio del método de componentes principales con una rotación varimax. Se extrajeron cuatro factores por tener autovalores mayores a uno. La asignación de los reactivos a cada factor se realizó con base en dos criterios: que el reactivo estuviera conceptualmente relacionado con el factor considerado, y que tuviera un peso factorial mayor a 0.35 en el factor correspondiente. Se eliminaron 18 reactivos que no cumplieron con alguno de los criterios mencionados. De esta manera, el cuestionario quedó conformado por 44 reactivos. Los cuatro factores extraídos explican el $43.17 \%$ de la varianza total. El primer factor agrupó a un total de 19 reactivos que explican el $18.68 \%$ de la varianza; esos reactivos se refieren al grado de contención o control de sí mismo, por lo que se le denominó Dominio de sí mismo. El segundo factor quedó compuesto por 12 reactivos que explican el 10.84\% de la va- 
Cuadro 1. Estructura factorial del Inventario de Ajuste de Weinberger

\begin{tabular}{|c|c|c|c|c|c|}
\hline \multirow{2}{*}{\multicolumn{2}{|c|}{ Reactivo }} & \multicolumn{4}{|c|}{ Peso factorial } \\
\hline & & \multirow{2}{*}{$\frac{\text { Factor I }}{.654}$} & \multirow[t]{2}{*}{ Factor II } & \multirow[t]{2}{*}{ Factor III } & \multirow[t]{2}{*}{ Factor IV } \\
\hline r66 & Si alguien hace algo que realmente no me gusta, le grito reclamándole. & & & & \\
\hline r68 & Cuando estoy enojado pierdo el control y permito que la gente lo note. & .602 & & & \\
\hline r73 & Molesto a la gente que me cae mal. & .640 & & & \\
\hline r80 & Ofendo a las personas que me molestan. & .725 & & & \\
\hline r84 & Cuando alguien me provoca, contesto a la provocación. & .634 & & & \\
\hline r48 & Hago las cosas sin prestarles mucha atención. & .543 & & & \\
\hline r54 & "Enloquezco" y hago cosas que a los demás puede no gustarles. & .700 & & & \\
\hline r57 & $\begin{array}{l}\text { Cuando hago algo por diversión (por ejemplo, ir de fiesta, actuar tontamente), tien- } \\
\text { do a seguirme de largo y llegar a los excesos. }\end{array}$ & .626 & & & \\
\hline r63 & $\begin{array}{l}\text { Me gusta hacer cosas nuevas y diferentes aunque la mayoría de la gente considera- } \\
\text { ría raras o no muy seguras. }\end{array}$ & .374 & & & \\
\hline r71 & Hago cosas que sé que no están del todo bien. & .607 & & & \\
\hline r20 & Hago más cosas fuera de la ley que la mayoría de la gente. & .603 & & & \\
\hline r49 & $\begin{array}{l}\text { Cuando se me presenta la oportunidad, tomo lo que quiero aunque no me perte- } \\
\text { nezca. }\end{array}$ & .634 & & & \\
\hline r55 & Hago cosas que en realidad son injustas para las personas que no me importan. & .725 & & & \\
\hline r56 & Hago trampa cuando sé que nadie se dará cuenta. & .625 & & & \\
\hline r60 & Rompo las leyes y las reglas con las que no estoy de acuerdo. & .658 & & & \\
\hline r72 & Digo lo primero que se me viene a la mente sin detenerme a pensarlo. & .528 & & & \\
\hline r76 & Si la gente hace cosas sin pedirme que los acompañe me siento rechazado. & .448 & & & \\
\hline r50 & Si alguien trata de herirme, me aseguro de desquitarme. & .683 & & & \\
\hline r53 & Me siento de tan mal humor que sólo me detengo y no hago nada. & -.505 & & & \\
\hline r42 & Con frecuencia me siento triste o desgraciado. & & .575 & & \\
\hline r69 & Me siento tan desanimado e infeliz que nada me hace sentir mejor. & & .464 & & \\
\hline r09 & Me siento inseguro de mí mismo. & & .472 & & \\
\hline r21 & En realidad me desagrado. & & .568 & & \\
\hline r27 & A veces me siento tan mal conmigo mismo que me gustaría ser alguien más. & & .532 & & \\
\hline r44 & Generalmente siento que soy el tipo de persona que quiero ser. & & .673 & & \\
\hline rO1 & Disfruto de la mayoría de las cosas que hago en la semana. & & .541 & & \\
\hline r07 & No importa qué esté haciendo, normalmente me la paso bien. & & .575 & & \\
\hline r15 & En general me considero una persona feliz. & & .687 & & \\
\hline r22 & Generalmente me la paso bien cuando hago cosas en compañía de otras personas. & & .434 & & \\
\hline r28 & Soy del tipo de personas que sonríe y ríe mucho. & & .552 & & \\
\hline r34 & Soy del tipo de personas que se divierte mucho. & & .694 & & \\
\hline r12 & $\begin{array}{l}\text { Recuerdo alguna ocasión en la que me he sentido tan enojado con alguien que he } \\
\text { sentido ganas de hacerle daño. }\end{array}$ & & & .416 & \\
\hline r16 & He hecho cosas que son incorrectas y de las que me he arrepentido después. & & & .600 & \\
\hline r29 & De vez en cuando me expreso mal de los demás a sus espaldas. & & & .532 & \\
\hline r30 & De vez en cuando rompo las promesas que he hecho. & & & .588 & \\
\hline r39 & $\begin{array}{l}\text { Hay ocasiones en las que no permito que la gente se entere de que he hecho algo } \\
\text { malo. }\end{array}$ & & & .387 & \\
\hline r37 & Hay ocasiones en las que no termino las cosas porque pierdo mucho el tiempo. & & & .519 & \\
\hline rO2 & En ocasiones me propongo hacer algo pero al final realizo otra cosa. & & & .586 & \\
\hline r79 & Me detengo a pensar las cosas antes de actuar. & & & & .648 \\
\hline r47 & Pienso en los demás antes de hacer algo que no les guste. & & & & .611 \\
\hline r51 & Disfruto haciendo algo por los demás, incluso aunque no reciba nada a cambio. & & & & .477 \\
\hline r59 & Me aseguro que haciendo lo que quiero no le causaré problemas a nadie. & & & & .541 \\
\hline r65 & Antes de hacer algo, pienso en que no afecte a la gente de mi alrededor. & & & & .655 \\
\hline r77 & Me esfuerzo mucho en no herir los sentimientos de los demás. & & & & .709 \\
\hline
\end{tabular}


rianza, y se refieren a la dimensión afectiva o la percepción de distrés emocional, por lo que se les nombró Experiencia subjetiva de distrés. El tercer factor consta de siete reactivos que explican el $7.10 \%$ de la varianza; tales reactivos se refieren a la actitud defensiva, por lo que se nombraron Defensividad. Por último, el cuarto factor agrupó a seis reactivos que explican el $6.54 \%$ de la varianza, e indican el grado de preocupación o consideración por otros, por lo que se les llamó Consideración hacia los demás. Los tres primeros factores recibieron el mismo nombre que los factores del instrumento original. Sin embargo, el cuarto factor contiene reactivos que en el instrumento original en inglés se agrupan dentro de la subescala con el mismo nombre (Consideración hacia los demás), perteneciente al factor Dominio de sí mismo. En el cuadro 1 se presentan los 44 reactivos del cuestionario final, así como sus pesos factoriales.

Finalmente, para evaluar la confiabilidad del cuestionario, se obtuvieron los coeficientes alpha de Cronbach de cada uno de los factores, así como el de la escala general. Los resultados mostraron una consistencia interna satisfactoria y se muestran en el cuadro 2.

\section{CONCLUSIONES}

El Inventario de Weinberger es una herramienta que evalúa dos aspectos centrales del ajuste socioemocional: la dimensión de la experiencia subjetiva de distrés, que proporciona una medida general de las tendencias de los individuos a sentirse insatisfechos con ellos mismos y con su capacidad para lograr los resultados deseados; y la dimensión de la contención (es decir, el dominio de sí mismo), que abarca variables socioemocionales relacionadas con la socialización y el autocontrol, y se refiere a la supresión de los deseos egoístas a corto plazo a cambio del interés por los demás a largo plazo. ${ }^{27}$ Además, aunque en los detalles difieren considerablemente, la conceptualización de distrés y contención como dos dimensiones amplias y relativamente estables de ajuste es paralela a la construcción de la ego-resiliencia y del ego-control. ${ }^{41}$

En este sentido, el inventario es un buen instrumento para medir el ajuste socioemocional y las dimensiones y subdimensiones de la personalidad de manera eficiente $\mathrm{y}$

Cuadro 2. Índices de consistencia interna del Inventario de Ajuste de Weinberger

\begin{tabular}{lcc}
\hline & $\begin{array}{c}\text { Número } \\
\text { de reactivos }\end{array}$ & Alfa \\
\hline Factor I Dominio de sí mismo & 12 & .89 \\
Factor II Experiencia subjetiva de distrés & 19 & .84 \\
Factor III Defensividad & 7 & .69 \\
Factor IV Consideración hacia los demás & 6 & .74 \\
Cuestionario total & 44 & .78 \\
\hline
\end{tabular}

confiable, ya que la estructura factorial de las escalas de distrés y contención es comparable en la preadolescencia y en la edad avanzada, tanto en poblaciones clínicas como normales.

Los resultados que se obtuvieron muestran que mantiene sus características de consistencia interna, con una estructura factorial conceptualmente congruente. Además, la reducción a 44 reactivos hace que el inventario sea más manejable en su aplicación.

La estructura factorial del Inventario de Weinberger quedó conformada de manera similar a los factores originales. No obstante, la subdimensión Consideración hacia los demás, que en la versión original formaba parte del factor Control de sí mismo, conformó un cuarto factor en la versión en español. Los reactivos de este factor fueron "Pienso en los demás antes de hacer algo que no les guste (r47)", "Disfruto haciendo algo por los demás, incluso aunque no reciba nada a cambio (r51)", "Me aseguro que haciendo lo que quiero no le causaré problemas a nadie (r59)", "Antes de hacer algo, pienso en que no afecte a la gente de mí alrededor (r65)", "Me esfuerzo mucho por no herir los sentimientos de los demás (r77)" y "Me detengo a pensar las cosas antes de actuar (r79)". Esto parece ser un indicador de diferencias culturales importantes en la población mexicana, aunque no se descartan otros elementos de base. Por ejemplo, Weinberger postula que el control de sí mismo abarca cuatro elementos que son guiados por metas intrapersonales (control de los impulsos), interpersonales (supresión de la agresión y consideración hacia los demás) y comunales (responsabilidad), y las ha validado como un solo factor. Sin embargo, Farrel y Sullivan $^{40}$ sugieren que la subescala de consideración hacia los demás no debería incluirse dentro del control de sí mismo o contención. Esto porque, de acuerdo con la tipología de la personalidad, mientras que el control de los impulsos y la responsabilidad reflejan autocontrol o moralidad, la consideración hacia los demás se asocia más con amabilidad. De hecho, éstos y otros autores han reexaminado la estructura factorial de esta subescala y han encontrado diferencias como las que se muestran aquí. ${ }^{40,42}$

Asimismo, el reactivo "Me siento de tan mal humor que sólo me detengo y no hago nada (r53)", que se ubicó en el factor Dominio de sí mismo, estaba antes en Experiencia subjetiva de distrés. Nuevamente, esta variación puede ser producto de diferencias culturales respecto a cómo se perciben las emociones y cómo se actúa ante ellas.

Respecto a la posible utilidad de este cuestionario, ya se ha mostrado previamente que el Inventario de Weinberger es una herramienta útil para la investigación en el área de la psicología social, de la salud mental y de la salud pública, pues permite evaluar la inhibición emocional como un aspecto importante del malestar emocional de los individuos. Además, su uso ha permitido determinar la magnitud de la problemática en poblaciones diversas, así como el abordaje al instrumentar acciones de prevención y tratamiento. Por 
lo anterior, la traducción y validación de este instrumento en población mexicana abrirá el campo de investigación en esta población y en otras de habla hispana, y permitirá las comparaciones entre poblaciones. Finalmente, el cuestionario en español se ha reducido sustancialmente en el número de reactivos, por lo que su aplicación y análisis resulta más fácil y permite la aplicación de otros instrumentos que amplíen o confirmen su utilidad.

\section{AGRADECIMIENTOS}

Agradecemos a la doctora Janine Giese-Davis, del Department of Oncology de la Facultad de Medicina de la University of Calgary, quien nos facilitó amablemente la versión en inglés del Inventario de Weinberger, y a la maestra Lorena Amelia Mercado Lara, de la Facultad de Idiomas de la Universidad Veracruzana, quien realizó la traducción y retraducción del instrumento. También agradecemos el apoyo técnico de las estudiantes de licenciatura Elizabeth Vázquez Montero, Liliana Yépez Olvera y Raquel González Ochoa, quienes aplicaron los cuestionarios y capturaron los datos. Este proyecto fue financiado gracias a los recursos del Instituto de Investigaciones Biológicas y la Facultad de Enfermería, Región Veracruz, de la Universidad Veracruzana.

\section{REFERENCIAS}

1. Baumeister RF, Tice DM. Emotion and self-presentation. En: Hogan $\mathrm{R}$, Jones WH (eds.). Perspectives in personality. Greenwich, CT: JAI Press; 1987; vol. 2; pp.181-199.

2. Jiménez B. Psicología de la personalidad. Procesos. Madrid: Thompson; 2007; pp.203-213.

3. Harris PL. Children and emotion: The development of psychological understanding. New York: Basil Blackwell; 1989.

4. Henao López GC, García Vesga MC. Interacción familiar y desarrollo emocional en niños y niñas. Revista Latinoamericana Ciencias Socials 2009.

5. Saarni C, Crowley M. The development of emotion regulation: Effects on emotional state and expression. En: Blechman EA (ed.). Emotions and the family: For better or for worse. Hillsdale, NJ: Lawrence Erlbaum; 1990; pp.53-73.

6. Retana-Franco BE, Sánchez-Aragón R. Rastreando en el pasado... formas de regular la felicidad, la tristeza, el amor, el enojo y el miedo. Universitas Psychologica 2009;9(1):179-197.

7. King LA, Emmons RA. Conflict over emotional expression: Psychological and physical correlates. J Personality Social Psychology 1990;58:864-877.

8. Singer J. Personality and psychoteraphy: Treating the whole person. New York: The Guilford Press; 2005.

9. Caspi A, Elder GH, Bem DG. Moving against the world: Life-course patterns of explosive children. Developmental Psychology 1987;23:308-313.

10. Larson DG, Chastain R. Self-concealment: Conceptualization, measurement, and health implications. J Social Clinical Psychology 1990;9:439-455.

11. Pennebaker JW. Confession, inhibition, and disease. En: Berkowitz L (ed.). Advances in experimental social psychology. Orlando: Academic Press; 1989; vol. 22. pp.211-244.

12. Barra E. Influencia del estado emocional en la salud física. Terápia Psicológica 2003;21(1):55-60.

13. Weinberger DA. The construct validity of the repressive coping style. En: Singer JL (ed.). Repression and dissociation: Implications for per- sonality theory, psychopathology, and health. Chicago: University of Chicago Press; 1990; pp.337-386.

14. Weinberger DA, Schwartz GE. Distress and restraint as superordinate dimensions of self-reported adjustment: A typological perspective. J Personality 1990;58:381-417.

15. Wilson JJ, Willams SE, Garner E, Duxbury E et al. Personality traits in juvenile delinquents: Associations with peer and family relations. Jefferson J Psychiatry 2001;16(1):32-45.

16. Shedler J, Mayman M, Manis M. The illusion of mental health. American Psychologist 1993;48:1117-1131.

17. Asendorpf JB, Scherer KR. The discrepant repressor Differentiation between low anxiety, high anxiety, and repression of anxiety by autonomic-facial-verbal patterns of behavior. J Personality Social Psychology 1983;45:1334-1346.

18. King AC, Taylor CB, Albright CA, Haskells WL. The relationship between repressive and defensive coping styles and blood pressure responses in healthy, middle-aged men and women. J Psychosomatic Research 1990;34:461-471.

19. Weinberger DA, Schwartz GE, Davidson RJ. Low-anxious, highanxious, and repressive coping styles: Psychometric patterns and behavioral and physiological responses to stress. J Abnormal Psychology 1979;88:369-380.

20. Schwartz GE. Psychobiology of repression and health: A systems approach. En: Singer JL (ed.). Repression and dissociation: Implications for personality theory, psychopathology, and health. Chicago: University of Chicago Press; 1990; pp.405-434.

21. Phares E, Lamiell JT. Personahty. Annual Review Psychology 1977;28: 113-140.

22. Rorer LG, Widiger TA. Personality structure and assessment. Annual Review Psychology 1983;34:431-463.

23. London H, Exner JE. Dimensions of personality. New York: Wiley; 1978.

24. Eysenck HJ, Eysenck MW. Personality and individual differences. A natural science approach. New York: Plenum Press; 1985.

25. Hampson SE, John OP, Goldberg LR. Category breadth and hierarchical structure in personality Studies of asymmetries $\mathrm{m}$ judgments of trait implications. J Personality Social Psychology 1986;51:37-54.

26. McCrae RR, Costa PT Jr. Validation of the five-factor model of personality across instruments and observers. J Personality Social Psychology 1987;52:81-90.

27. Weinberger DA, Feldman SS, Ford ME. Social-emotional adjustment in older children and adults 11 External validation of subdimensions of distress and restraint. (1989). Manuscript submitted for publication.

28. Tellegen A. Stmctures of mood and personality and their relevance to assessing anxiety, with an emphasis on self-report. En: Tuma AH, Maser J (eds.). Anxiety and the anxiety disorders. Hillsdale, NJ: Lawrence Erlbaum; 1985; pp.681-706.

29. Digman JM. Five robust trait dimensions Development, stability, and utility. J Personality 1989;57:195-214.

30. Weinberger DA. Distress and self-restraint as measures of adjustment across the life span: Confirmatory factor analyses in clinical and nonclinical samples. Psychological Assessment 1997;9(2):132-135.

31. Huckaby W, Kohler M, Garner EH, Steiner H. A comparison of the Weinberger adjustment inventory and the Minnesota multiphasic personality inventory with incarcerated adolescent males. Child Psychiatry Human Development 1998;28:273-286.

32. Weinberger DA. Social-emotional adjustment in older children and adults: I. Psychometric properties of the Weinberger adjustment inventory, 1990b, Unpublished Manuscript.

33. Giese-Davis J, Spiegel D. Suppression, repressive-defensiveness, restraint, and distress in metastatic breast cancer: Separable or inseparable constructs? J Personality 2001;69:417-449.

34. Turvey C, Salovey P. Measures of repression: converging on the same construct? Imagination Cognition Personality 1993-94;13(4):279-289.

35. Giese-Davis J, Conrad A, Nouriani B, Spiegel D. Exploring emotionregulation and autonomic physiology in metastatic breast cancer pa- 
tients: Repression, suppression, and restraint of hostility. Pers Individ Dif 2008;44(1):226-237.

36. McKenna MC, Zevon MA, Corn B, Rounds J. Psychosocial factors and the development of breast cancer: A meta-analysis. Health Psychology 1999;18:520-531.

37. Jensen MR. Psychobiological factors predicting the course of breast cancer. J Personality 1987;55:317-342.

38. Anastasi A, Urbina S. Test psicológicos. España: Aguilar; 2000.

39. Nunnally JC. Teoría psicométrica. México: Trillas; 2000.
40. Farrell AD, Sullivan TN. Structure of the Weinberger adjustment inventory self-restraint scale and its relation to problem behaviors in adolescence. Psychological Assessment 2000;12:394-401.

41. Block JH, Block J. The role of ego-control and ego-resiliency in the organization of behavior. En: Collins WA (ed). Minnesota symposia on child psychology. Hillsdale, NJ: Lawrence Erlbaum; 1980; vol 13; pp.9-101.

42. Sumter SR, Bokhorst CL, Westenberg PM. The Robustness of the Factor Structure of the Self-Restraint Scale: What does self-restraint encompass. J Research Personality 2008;42:1082-1087.

Artículo sin conflicto de intereses 